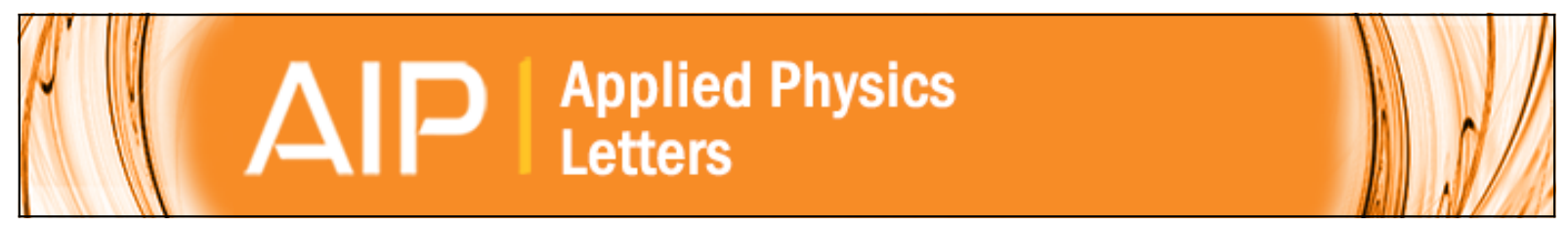

\title{
Predictive model for scanned probe oxidation kinetics
}

J. A. Dagata, F. Perez-Murano, G. Abadal, K. Morimoto, T. Inoue, J. Itoh, and H. Yokoyama

Citation: Applied Physics Letters 76, 2710 (2000); doi: 10.1063/1.126451

View online: http://dx.doi.org/10.1063/1.126451

View Table of Contents: http://scitation.aip.org/content/aip/journal/apl/76/19?ver=pdfcov

Published by the AIP Publishing

\section{Instruments for advanced science}
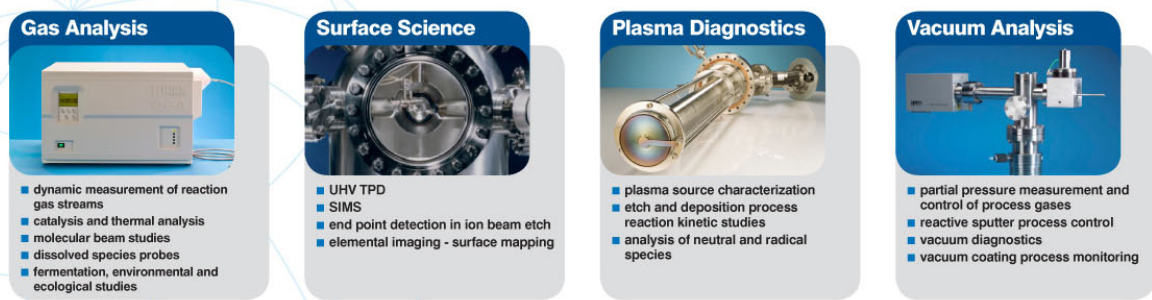

contact Hiden Analytical for further details

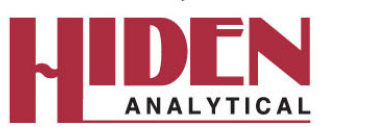

info@hideninc.com www.HidenAnalytical.com CLICK to view our product catalogue 


\title{
Predictive model for scanned probe oxidation kinetics
}

\author{
J. A. Dagata ${ }^{\text {a) }}$ \\ National Institute of Standards and Technology, Gaithersburg, Maryland 20899 \\ F. Perez-Murano and G. Abadal \\ Department d'Enginyeria Electronica, Universitat Autonoma de Barcelona, Barcelona, Spain \\ K. Morimoto \\ Matsushita Electrical Industrial Co., LTD, Osaka, Japan
}

T. Inoue, J. Itoh, and H. Yokoyama

Electrotechnical Laboratory, Tsukuba, Japan

(Received 24 January 2000; accepted for publication 13 March 2000)

\begin{abstract}
Previous descriptions of scanned probe oxidation kinetics involved implicit assumptions that one-dimensional, steady-state models apply for arbitrary values of applied voltage and pulse duration. These assumptions have led to inconsistent interpretations regarding the fundamental processes that contribute to control of oxide growth rate. We propose a model that includes a temporal crossover of the system from transient to steady-state growth and a spatial crossover from predominantly vertical to coupled lateral growth. The model provides an excellent fit of available experimental data. (C) 2000 American Institute of Physics. [S0003-6951(00)03119-3]
\end{abstract}

For scanned probe microscope (SPM) oxidation ${ }^{1}$ to become established as a routine and reliable technique for nanodevice $^{2}$ and nanostructure ${ }^{3}$ fabrication, a predictive, quantitative model embodying a realistic physical description of the growth mechanism is essential. There has been considerable recent interest in interpreting experimental kinetic data for SPM oxidation ${ }^{4-12}$ and general agreement has emerged on the dependence of oxide height, $h(t)$, width, $W(t)$, and aspect ratio, $h / W$, on exposure parameters, i.e., voltage applied between SPM tip and substrate, $V$ $[\approx 5-20 \mathrm{~V}]$, and the pulse duration, $t\left[\approx 10^{-3}-10^{3} \mathrm{~s}\right]$. In 1995 Gordon $^{7}$ suggested that Cabrera-Mott theory ${ }^{13}$ was appropriate, with significant progress in this direction reported in 1997 by Stievenard ${ }^{8}$ and Avouris. ${ }^{9}$ Stievenard arrived at the inverse- $\log$ form, $1 / h(t)=k(V)-\log t$, beginning with the Cabrera-Mott assumptions and by introducing a thickness-dependent cutoff field $E_{L}=V / h_{L}$. Avouris proposed a direct-log form, $h(t)=k^{\prime}(V) \cdot \log t$, instead. It is noteworthy that Stievenard and Avouris achieved apparently satisfactory empirical fits of their data based on these alternate forms of the growth, since each equation is derived from very different assumptions according to discussions of Fehlner and Mott. ${ }^{14}$

Recognizing that the direct-log form is the appropriate one for SPM oxidation, we examine the assumptions used to derive it. Uhlig ${ }^{15}$ obtained the direct-log form, $h(t)$ $=k^{\prime \prime}(V) \cdot \log \left(k_{U} t+1\right)$, by assuming that the rate-controlling step involves interaction of oxyanions with electronic species-holes or electrons-and defects at a metal-oxide interface (here, the $\mathrm{Si} / \mathrm{SiO}_{x}$ interface). In particular, Uhlig's model unifies key concepts recognized by Stievenard and Avouris with our conjectures about the influence of space charge on SPM oxide growth. According to this model, production of charged defects ${ }^{16}$ leads to a buildup of space

${ }^{a)}$ Electronic mail:john.dagata@nist.gov charge within the oxide, inhibiting further growth over long pulse times. ${ }^{10,12}$ If we follow Uhlig in attributing the thickness-related change in activation energy to space charge rather than stress as we have suggested in previous papers, we reach full agreement with Avouris on the form of the rate law for SPM oxide growth. We can also invoke Uhlig's theory to provide a physical basis for Stievenard's cutoff field, since $E_{L}$ is simply the potential at which space charge in the oxide nulls the externally applied field. As Uhlig has indicated, this is the point at which fast oxide growth (in the vertical dimension) ceases.

In 1995 Teuschler $^{6}$ demonstrated that an empirical power law, $h(t)=a_{0}\left(V-V_{\text {th }}\right) \cdot t^{\gamma}$, provides a remarkably good fit to the experimental data, with subsequent work indicating that $\gamma$ ranges from 0.12 to 0.4 . $\left[a_{0}\right.$ and $V_{\text {th }}$ are constants.] Fractional power laws have long been associated with universal relaxation phenomena. ${ }^{17-19}$ An exponential relaxation process, $\exp (-t / \tau)$, is "stretched out" over a longer time scale by a loss mechanism, so that $\exp (-t / \tau) \rightarrow \exp (-t / \tau)^{\gamma}$, with $0<\gamma \leqslant 1$. Since oxidation involves the transport of ionic species across the growing oxide film and subsequent reaction at one or the other of ambient/oxide and oxide/substrate interfaces, the process constitutes a reaction-diffusion system which may exhibit relaxation-dissipation behavior. In particular, note that Uhlig, in deriving the direct-log form did so by neglecting a second-order integration term of the Poisson equation justified on the assumption that $h(t) \ll h_{L}$. [C.f., Equations (7)(11) in Ref. 15.] Direct experimental evidence indicates that $h_{L}$ is on the order of 10 nanometers for SPM oxidation, with oxide thickness approaching or exceeding this value. ${ }^{8,10,12} \mathrm{~A}$ numerical integration of the linear, $\int \exp (a \cdot h) d h=\int d t$, and the quadratic expressions, $\int \exp \left(a \cdot h^{2}+b \cdot h+c\right) d h=\int d t$, indicates that addition of the quadratic term increases the time required to achieve a given thickness by a factor of 2.5 , thus $\gamma=(2.5)^{-1}$, or 0.4 , for a retarding potential. Substituting $t^{0.4}$ 
TABLE I. SPM oxidation kinetics represented as a set of integrated rate equations in the form derived by Alberty and Miller (Ref. 20). Notation for the individual reaction steps and rate constants are as they appear in Alberty-Miller, Equations (5), (16)-(8). Rate equations for species $A, B$, and $C$ are derived assuming that the initial concentrations are $A=A_{o}, B=0, C=0$. The reaction sequence symbolized by $(A \rightarrow B)$ is based upon the discussion of Poindexter.

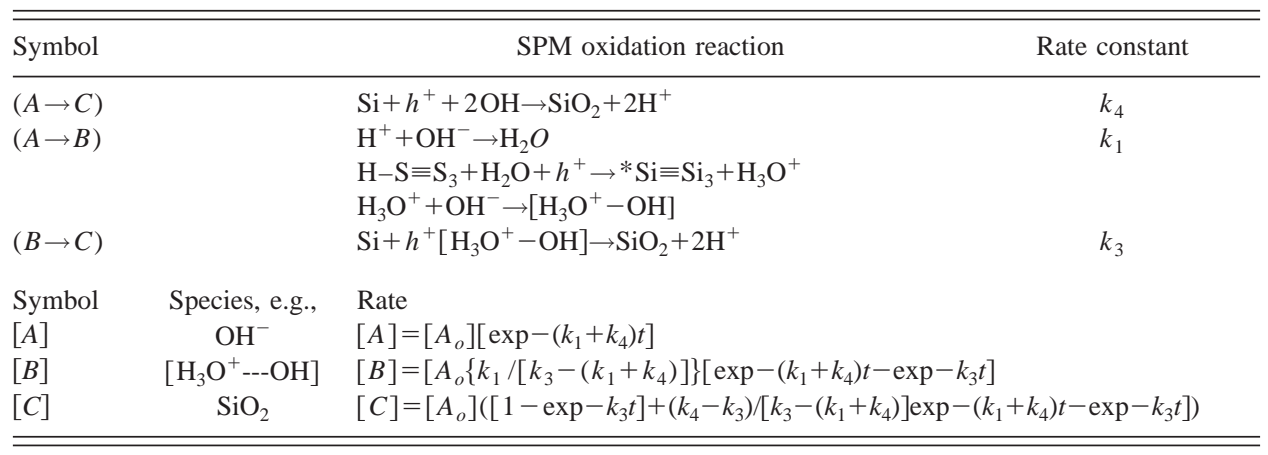

for $t$ in the Uhlig $\log$ form, $h(t)=\log \left(t^{0.4} / \tau+1\right)$, provides a quantitative fit of experimental SPM oxidation data, valid over eight time decades.

A single overall rate constant, $k_{U}=1 / \tau$, in the Uhlig expression does not provide us with sufficient insight into how oxyanions, trapping sites, and electronic species interact under varying SPM oxidation conditions. However, we can augment Uhlig's basic concept by introducing space charge buildup concepts. Let's assume that silicon oxidation consists of a "direct"' irreversible process, $A \rightarrow C$, and an "indirect' one, $A \rightarrow B \rightarrow C$, according to reactions and rate constants defined in Table I. (See the discussions of Jonscher ${ }^{17}$ and Mashkov ${ }^{19}$ for examples.) Integrated rate expressions for $A(t), B(t)$, and $C(t)$ have been published by Alberty and Miller ${ }^{20}$ and are also given in Table I. The time evolution of the reactant and product concentrations defined by the Alberty-Miller scheme is displayed in Fig. 1, upper panel. Reaction of an initial concentration of oxyanions is represented by $[A(t)]$. The density of fixed charge traps at the growing $\mathrm{Si} / \mathrm{SiO}_{x}$ interface and their dissipative effect on the oxidation rate is described by $[B(t)]$. Evolution of the $\mathrm{SiO}_{x}$ concentration, the symbol $[C(t)]$, exhibits a growth curve that is evidently shaped by competition between the direct and indirect oxidation processes. If the Alberty-Miller equations provide us with a useful model for SPM oxidation under nondissipative conditions, then for the actual conditions encountered during SPM oxidation, $h(t) \propto\left[C\left(t^{0.4} ; k_{i}\right)\right]$. The lower panel of Fig. 1 presents a comparison of AlbertyMiller, Uhlig, and Teuschler fits. Note that for $t<1 \mathrm{~s}$, all three expressions are equivalent.

Growth rates calculated using the Alberty-Miller and Uhlig (dot-dash) expressions are compared with experimental SPM oxidation data in Fig. 2, left-hand side. [Experimental SPM data in this figure are replotted from Avouris, Fig. 2(a).] In fitting experimental data, values for the rate constants are not arbitrarily chosen. An initial value for the steady-state rate constant $k_{3}$ was determined directly by fitting the long-time kinetic data, using Equation (278) of Fromhold. ${ }^{21}$ A satisfactory fit for the steady-state portion of the growth curve leads to an underestimate of oxide growth at short time, which can be subtracted from the experimental data to obtain an initial estimate of $k_{4}$. We can then figure out what $k_{1}$ ought to be by fitting the Alberty-Miller expression to the entire span of the experimental data. This leads to expected ordering of the magnitude of the rate constants, $k_{1}>k_{4}>k_{3}$, which says that initially the direct $(A \rightarrow C)$ oxidation process occurs, but that over time the trapped state $(A \rightarrow B \rightarrow C$ ) builds up and interferes with growth. From the linear-log oresentation in Fig. 2, upper panel, we see that the simple Uhlig direct-log function does not adequately describe the modulated growth observed in the experimental results at long times. The origin of these modulated features in terms of competing rates, becomes evident from a glance at how $[C(t)]$ evolves in Fig. 1. A survey of SPM oxidation literature yields numerous examples of previously overlooked modulation features in the published data-as long as a representative sampling of the steady-state regime is included. For gaining a fundamental understanding of SPM
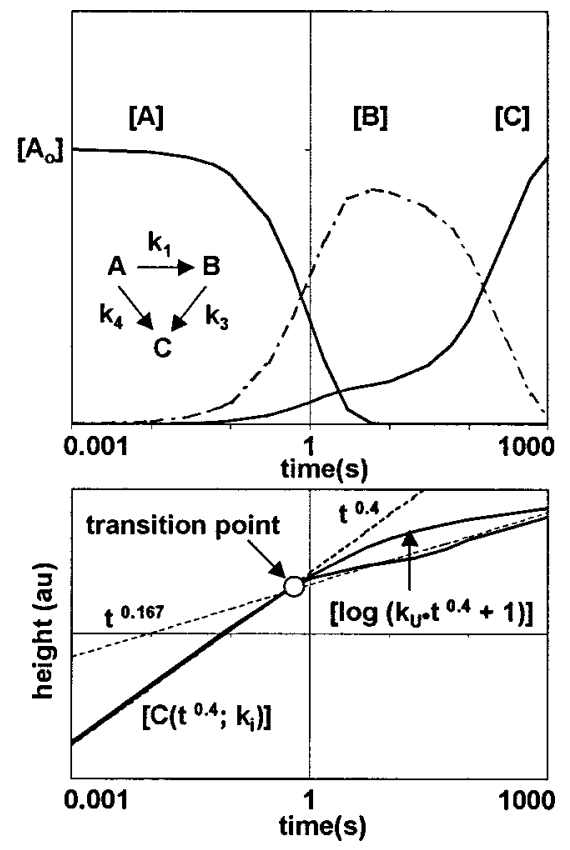

FIG. 1. Upper panel: SPM oxidation model based on the Alberty-Miller integrated rate equations. A direct pathway for reaction of oxyanions with silicon at the $\mathrm{Si} / \mathrm{SiO}_{2}$ interface is given by $(A \rightarrow C)$ and an indirect reaction pathway, mediated by trapped charge defects at the interface, is given by $(A \rightarrow B)$ followed by $(B \rightarrow C)$. Lower panel: $\log -\log$ plot of SPM oxide feature height vs pulse time comparing the Teuschler power law, Uhlig direct-log, and Alberty-Miller expressions. The Uhlig rate constant is $k_{U}$ $=0.5$ and the Alberty-Miller rate constants, as defined in Table I, are $k_{1}$ $=085, k_{3}=0.0035$, and $k_{4}=0.125$. Notice that all three forms exhibit identical power law behavior, $t^{0.4}$, in the transient regime, i.e., for $t \leqslant 500 \mathrm{~ms}$. 

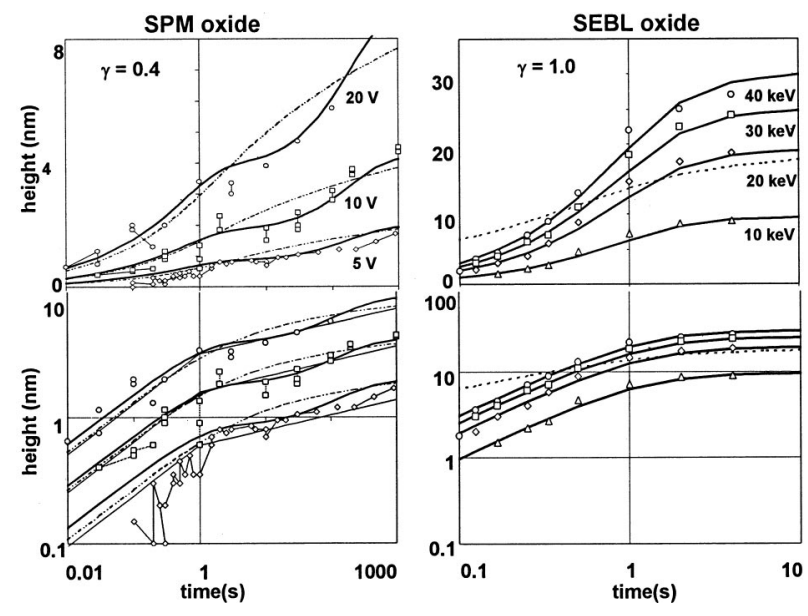

FIG. 2. Left-hand side: Calculated and experimental SPM oxidation kinetics. Data are presented in linear-log form in the upper panel, and in $\log -\log$ form in the lower panel. Exposure conditions are given in terms of applied bias voltage, $V_{\mathrm{dc}}=5,10$, and $20 \mathrm{~V}$, for pulse duration of $10^{-2}$ to $10^{3} \mathrm{~s}$ in contact mode. [Experimental SPM data, open circles, etc., are taken from Avouris, Fig. 2(a) of Ref. 9.] SPM growth rates, calculated using the Alberty-Miller expression with $\gamma=0.4$, (heavy solid line) and the Uhlig direct-log form (dot-dash line) are compared to power-law curves (light solid lines). Although both follow the transition from transient to steadystate growth, only the Alberty-Miller form reproduces the modulated growth observed in the steady-state regime, i.e., for $t>1 \mathrm{~s}$. Right-hand side: Calculated and experimental SEBL oxidation kinetics. Exposure conditions are given in terms of electron accelerating voltage, $V_{\text {acc }}=10,20,30$, and 40 $\mathrm{keV}$, for dwell times of $10^{-1}$ to $10 \mathrm{~s}$. [Experimental data, open circles, etc., are taken from Wei, Fig. 2(b) of Ref. 12.] The Alberty-Miller expression with $\gamma=1.0$ (solid line), is shown in comparison to a representative curve calculated with $\gamma=0.4$ (dashed line).

oxidation, the transition point (or region) is an essential concept. In Fig. 2 a transition from transient growth, characterized by a $t^{0.4}$ power-law dependence, to steady-state growth, characterized by a $t^{0.167}$ dependence, is clearly observed.

Figure 2, right-hand side, presents calculated and recently published experimental oxide growth kinetics for scanning electron-beam lithography (SEBL) oxidation. ${ }^{12} \mathrm{Al}-$ though exposure conditions for SPM and SEBL are vastly different, similarity of the characteristic self-limiting kinetics suggests generation of charged defects appears to be a common control factor for both processes. ${ }^{10,12}$ [Experimental SEBL data in this figure are replotted from Wei, Fig. 2(b) of Ref. 12.] The Alberty-Miller expression, with $\gamma=1.0$ (solid line), is shown in comparison to a representative curve calculated with $\gamma=0.4$ (dashed line) which is the dilation factor used for SPM oxidation. These results give us a degree of confidence that the Alberty-Miller equations describe the most significant aspects of silicon oxidation, at least for low temperatures. (See Wolters ${ }^{18}$ for a somewhat related approach to thermal oxidation of silicon.)

For the fits of experimental SPM and SEBL kinetic data reported here, we have emphasized only voltage and time dependence of the oxide growth rate. Geometrical factors, the SPM tip radius-of-curvature, relative humidity, and tipsubstrate distance, for instance, are not described. These factors may weakly affect the relative magnitude of the rate constants $k_{i}$. We have evaluated all relevant SPM kinetic data in an effort to quantitatively model the subtle, yet significant, [three-dimensional (3D)] differences in oxidation kinetics as a function of scan speed, voltage modulation, and contact versus noncontact modes. These results will be reported elsewhere. Figure 2 should make it clear, however, that future efforts to interpret SPM oxidation kinetics data must include a sufficient range of time and voltage, along with additional factors like tip radius and humidity, in order that the true functional dependence of the growth rate may be described correctly.

The authors thank Y. Y. Wei (ORNL) for making the SEBL data available to us in numerical form, and acknowledge pertinent discussions with E. Dubois (IEMN Lille), E. S. Snow (NRL), and R. Garcia (IMM, Madrid) on various aspects of SPM oxidation kinetics. Financial support from Fulbright comission (project 99202) is acknowledged. This work was performed in part under management of FED as part of the Quantum Functional Devices Project in Japan.

${ }^{1}$ J. A. Dagata, J. Schneir, H. H. Harary, C. J. Evans, M. T. Postek, and J. Bennett, Appl. Phys. Lett. 56, 2001 (1990); J. A. Dagata, Science 270, 1625 (1995), and references therein.

${ }^{2}$ K. Matsumoto, Y. Gotoh, T. Maeda, J. A. Dagata, and J. S. Harris, Appl. Phys. Lett. 76, 239 (1999).

${ }^{3}$ R. W. Cohn, S. F. Lyuksyutov, K. M. Walsh, and M. M. Crain, Opt. Rev. 6, 345 (1999); G. Abadal, A. Boisen, Z. J. Davis, O. Hansen, and F. Grey, Appl. Phys. Lett. 74, 3206 (1999).

${ }^{4}$ E. S. Snow and P. M. Campbell, Appl. Phys. Lett. 64, 1932 (1994).

${ }^{5}$ T. Hattori, Y. Ejiri, K. Saito, and M. Yasutake, J. Vac. Sci. Technol. A 12, 2586 (1994)

${ }^{6}$ T. Teuschler, K. Mahr, S. Miyazaki, M. Hundhausen, and L. Ley, Appl. Phys. Lett. 67, 3144 (1995).

${ }^{7}$ A. E. Gordon, R. T. Fayfield, D. D. Litfin, and T. K. Higman, J. Vac. Sci. Technol. B 13, 2805 (1995).

${ }^{8}$ D. Stievenard, P. A. Fontaine, and E. Dubois, Appl. Phys. Lett. 70, 3272 (1997).

${ }^{9}$ P. Avouris, T. Hertel, and R. Martel, Appl. Phys. Lett. 71, 285 (1997).

${ }^{10}$ J. A. Dagata, T. Inoue, J. Itoh, and H. Yokoyama, Appl. Phys. Lett. 73, 271 (1998); J. A. Dagata, T. Inoue, J. Itoh, K. Matsumoto, and H. Yokoyama, J. Appl. Phys. 84, 6891 (1998); F. Pérez-Murano, K. Morimoto, and J. A. Dagata, Appl. Phys. Lett. 75, 199 (1999); K. Morimoto, F. Perez-Murano, and J. A. Dagata, Appl. Surf. Sci. (in press).

${ }^{11}$ R. Garcia, M. Calleja, and H. Rohrer, J. Appl. Phys. 86, 1898 (1999).

${ }^{12}$ Y. Y. Wei and G. Eres, Appl. Phys. Lett. 76, 194 (2000).

${ }^{13}$ N. Cabrera and N. F. Mott, Rep. Prog. Phys. 12, 163 (1948).

${ }^{14}$ F. P. Fehlner and N. F. Mott, Oxid. Met. 2, 59 (1970); F. P. Fehlner, Philos. Mag. B 55, 633 (1987).

${ }^{15}$ H. H. Uhlig, Acta Metall. 4, 541 (1956).

${ }^{16}$ E. H. Poindexter, C. F. Young, and G. J. Gerardi, in Fundamental Aspects of Ultrathin Dielectrics on Si-based Devices, edited by E. Garfunkel, E. Gusev, and A. Vul' (Kluwer, Dordrecht, 1998), p. 300.

${ }^{17}$ A. K. Jonscher, Universal Relaxation Law (Chelsea Dielectrics Pr., London, 1996).

${ }^{18}$ D. R. Wolters and A. T. A. Zegers-van Duynhoven, J. Appl. Phys. 65, 5126 (1989)

${ }^{19}$ V. A. Mashkov, Wm. P. Austin, L. Zhang, and R. G. Leisure, Phys. Rev. Lett. 76, 2926 (1996).

${ }^{20}$ R. A. Alberty and W. G. Miller, J. Chem. Phys. 26, 1231 (1957); see also, S. W. Benson, The Foundations of Chemical Kinetics (McGraw-Hill, New York, 1960).

${ }^{21}$ T. Fromhold, Jr., in Oxides and Oxide Films, edited by J. W. Diggle and A. K. Vijh (Marcel Dekker, New York, 1976), Vol. 3, p. 1. 